\title{
Hearing difficulties, ear-related diagnoses and sickness absence or disability pension - a systematic literature review
}

\author{
Emilie Friberg*, Klas Gustafsson and Kristina Alexanderson
}

\begin{abstract}
Background: Hearing difficulties is a large public health problem, prognosticated to be the ninth leading burden of disease in 2030, and may also involve large consequences for work capacity. However, research regarding sickness absence and disability pension in relation to hearing difficulties is scarce. The aim was to gain knowledge about hearing difficulties or other ear-related diagnoses and sickness absence and disability pension through conducting a systematic literature review of published studies.

Methods: Studies presenting empirical data on hearing difficulties or ear-related diagnoses and sick leave or disability pension, published in scientific peer-reviewed journals, were included. Studies were sought for in three ways: in literature databases (Pub-Med, Embase, Psyclnfo, SSCl, and Cochrane) through March 2011, through scrutinising lists of references, and through contacts. Identified publications were assessed for relevance and data was extracted from the studies deemed relevant.

Results: A total of 18 studies were assessed as relevant and included in this review, regardless of scientific quality. Fourteen studies presented empirical data on hearing difficulties/ear diagnoses and sick leave and six on these conditions and disability pension. Only two studies presented rate ratios or odds ratios regarding associations between hearing difficulties and sick leave, and only two on hearing difficulties and risk of disability pension. Both measures of hearing difficulties and of sick leave varied considerable between the studies.

Conclusions: Remarkably few studies on hearing difficulties in relation to sickness absence or disability pension were identified. The results presented in them cannot provide evidence for direction or magnitude of potential associations.
\end{abstract}

Keywords: Sick leave, Disability pension, Hearing difficulties, Systematic literature review

\section{Background}

Hearing difficulties is a major public health concern [15], estimated to be the ninth leading burden of disease worldwide in the year 2030 [4]. Partial or complete hearing loss as well as other ear-related diagnoses such as vertigo or tinnitus can have consequences for work capacity. Sick leave or permanent marginalization from the labour market in terms of disability pension due to these diagnoses are, however, not much discussed or studied, although interventions regarding this are warranted and need to be based on scientific knowledge [6].

\footnotetext{
* Correspondence: Emilie.Friberg@ki.se

Division of Insurance Medicine, Department of Clinical Neuroscience, Karolinska Institutet, Stockholm SE-171 77, Sweden
}

Known causes of hearing difficulties include various factors such as age, noise exposure, heredity, infections, health, and socio-economic factors $[7,8]$. Hearing difficulties as well as other ear-related diagnoses such as tinnitus, may lead to increased levels of stress, partly through the limited possibilities to be involved in ordinary conversions and the constant strain to hear [9]. This could potentially affect the risk of sickness absence and/ or disability pension. Vertigo, balance related difficulties are also an important health problem that might lead to sick leave and exclusion from the labor market [10].

There are several indications of gender differences in the occurrence of hearing difficulties [9] as well as in sickness absence [11], why, gender stratified data regarding these aspects would be of interest.
C Biomed Central

(c) 2012 Friberg et al.; licensee BioMed Central Ltd. This is an Open Access article distributed under the terms of the Creative Commons Attribution License (http://creativecommons.org/licenses/by/2.0), which permits unrestricted use, distribution, and reproduction in any medium, provided the original work is properly cited. 
Sickness absence has a large impact on society, employers, work sites, as well as on the individual and his or her family $[6,12]$. As most individuals with a disease or an injury can work, sickness absence is seldom a good measure of morbidity on a population level [6], however, it is a good measure of the social consequences of morbidity in terms of work incapacity $[6,13]$. In order to take adequate measures to prevent marginalisation among people with hearing difficulties, knowledge regarding the magnitude of such sickness absence and disability pension, is needed. Therefore, the aim of this study was to establish the current knowledgebase on hearing difficulties and other ear-related diagnoses and sickness absence and/or disability pension though conducting a systematic literature review of such studies.

\section{Methods}

The systematic literature review was conducted in accordance to the PRISMA guidelines [14], in the following five steps; searches for studies, assessment of relevance of identified publications, data extraction, categorization of studies and synthesis of results from included studies. The following inclusion criteria were applied: Studies presenting empirical data on hearing difficulties or other ear-related diagnoses as well as on sick leave or disability pension published in scientific peer-reviewed journals. Both self-reported difficulties as well as ear-related diagnoses were included, to provide a wide inclusion and try to capture disorders and difficulties, which if diagnosed, would fall in the chapter of "Diseases of the ear and mastoid process" according to the "International Statistical Classification of Diseases and Related Health Problems, Tenth Revision" (ICD-10) [15].

Studies were sought for in the following three ways, no language or time restrictions were imposed:

1.) Searches, through March 2011, of the literature databases Pub-Med, Embase, PsycINFO, SSCI (Social Sciences Citation Index), and Cochrane using combinations of the following keywords: "sick leave" OR "sick-leave" OR "sickness absence" OR "sickness absent" OR "absenteeism" OR "return to work" OR "return-to-work" OR "disability pension" OR "work ability" OR "work inability" OR "work capacity" OR "work incapacity" OR "sickness benefit" OR "incapacity benefit" OR "work disability" OR "disability leave" OR "work disabled" AND "hearing" OR "deaf" OR "tinnitus" OR "vertigo" OR "auditory". The combination of the sick-leave and hearing terms gave a total of 1229 hits (duplicates included) in PubMed (sick-leave terms and: hearing 607; deaf 160: tinnitus 46; vertigo 96; auditory 320), 2436 in Embase (sick-leave terms and: hearing 1012; deaf
443; tinnitus 101; vertigo 300; auditory 580), 51 in PsycINFO (including book chapters) (sick-leave terms and: hearing 10; deaf 8; tinnitus 0; vertigo 0; auditory 33), 1101 in SSCI (sick-leave terms and: hearing 163; deaf 119; tinnitus 38; vertigo 49; auditory 732), and 737 in Cochrane (sick-leave terms and: hearing 225; deaf 15; tinnitus 46; vertigo 66; auditory 385).

2.) Scrutinising lists of references of all studies deemed relevant. This gave two additional studies.

3.) Through contacts, i.e. asking other researchers if they knew about possible relevant studies. One additional study was identified.

The searches were conducted independently by two persons (E. Friberg and K. Gustafsson). All identified publications were assessed for relevance according to the above inclusion criteria. Titles and abstracts were read independently by at least two authors. In case of uncertainty or disagreement, the full article was read and, if needed discussed until consensus was reached. All relevant studies were included, irrespective of their scientific quality. Information regarding study design, population, measures, data collection, analyses performed, and relevant results (where applicable, the most adjusted result was extracted) were extracted from the included studies. Thereafter, the studies were categorized with regard to study design, type of study group (i.e. population, employees, or patients), and outcome. Finally, results from the included studies were compiled.

\section{Results}

When excluding double hits from different databases, a total of 2663 publications were identified, of which 18 were assessed as relevant (Figure 1), that is, they disclosed data on hearing difficulties or other ear-related diagnoses and on sick leave [10,16-28] and/or disability pension [17,18,29-32] (Table 1). The included studies were conducted in eight different countries (Sweden, USA, UK, Germany, Netherland, France, Norway and Poland, Table 1). All included studies were published in English and only three were published before the year 2000 (Figure 2). The majority (56\%) of the studies had a cohort design, five (28\%) had a cross-sectional design, and three were intervention studies (Table 2).

There was considerable heterogeneity between the studies. Only four studies used population data, eight studies evaluated employed individuals, while six reported data on patients (Table 2). The hearing diagnoses/symptoms explored varied considerably between studies, including; sudden sensorineural hearing loss, otoaudiological diagnoses, unilateral vestibular loss, tinnitus, hearing impairment, Ménière's disease, otitis media, vestibular vertigo, post-lingual hearing loss, 


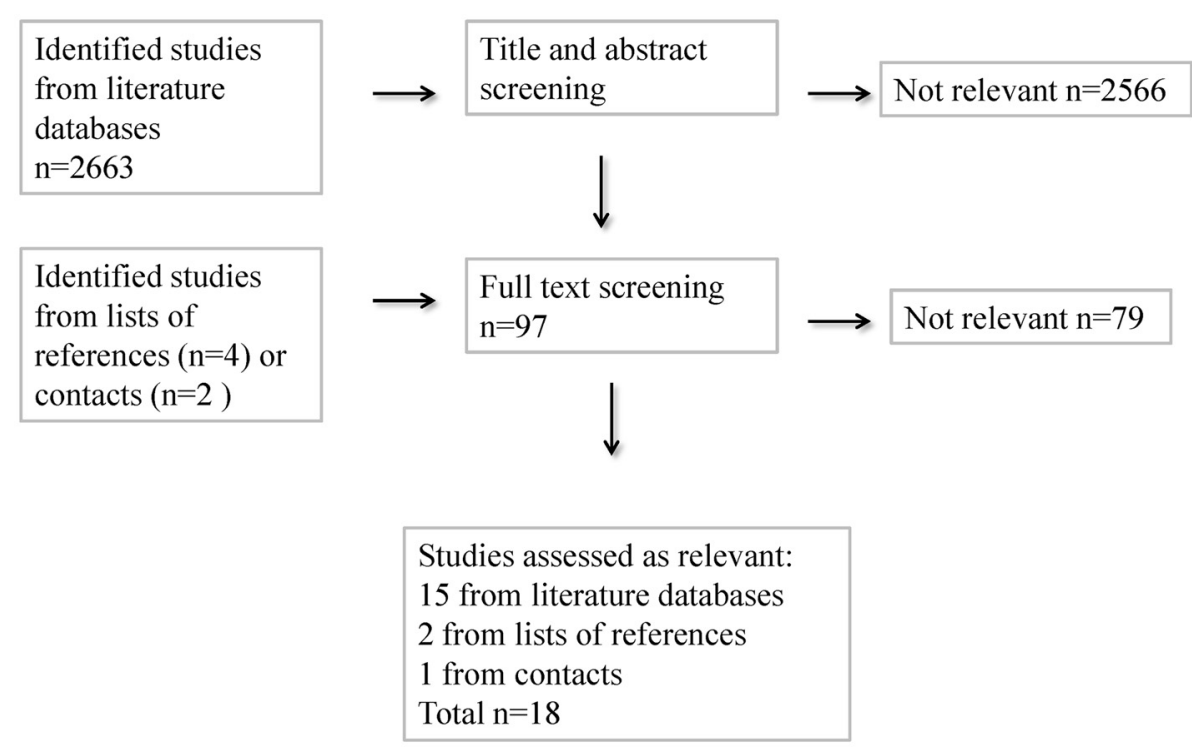

Figure 1 Number of identified publications and excluded, included, and relevant studies.

vertigo, and ear and mastoid diagnoses. Data on hearing difficulties were in many of the studies self reported $[10,16,19-21,28]$. Outcome variables in the studies were different types of measures of sick leave and disability pension. Only four studies presented associations (i.e. rate ratios or odds ratios) between hearing difficulties and sick leave $[20,23]$ or disability pension $[17,18]$, respectively. Five studies presented data for patient groups $[16,19,25-27]$ without any control or comparison group. Several studies were based on very small samples, for example, only six studies included more than 1000 individuals $[10,17,18,29,30,32]$ and five studies included less than 100 individuals [19,22,24-26] (Table 1). The mean number of participants was 3926. Only two studies presented data stratified by sex $[17,18]$ while six studies only included men $[23,25,28-31]$. A majority of the studies presented data for all-cause sick leave instead of diagnoses-specific sick leave whereas half of the studies on disability pension assessed diagnoses-specific disability pension (Table 3 ).

\section{Sickness absence}

In all, 45850 individuals were included in the studies presenting data on sickness absence, 44278 with known sex, of these $47 \%$ were men. The measures of sick leave were often self-reported, only in five studies were register data used [17,18,24,26,28].

Measures of sick leave varied considerably between studies, the following measures were used: proportion of patients on sick leave and grade (full or part time) of sick leave [16], sick leave $>7$ days due to otoaudiological diagnoses from national registers [17], number of individuals on long-term sick leave ( $>8$ weeks) due to vertigo or dizziness [18], sick leave yes/no [19,27], sick leave due to hearing difficulties: yes, no, do not remember [21], absent from work more than one month due to tinnitus [26], number of days and periods of sickness benefit [28], number of days having to stay at home, leave work, or cancel a planned schedule, being bedridden or largely incapacitated during that day [22], number of absence days during a 3-year period before and after treatment from the national registers [24], absence from work due to health or due to hearing impairment [25], reported sick leave among persons with dizziness [10], selfreported number of absence days in the last 12 months and reason for sick leave [20], sick leave more than 60 days reported by physician [23].

Both studies [20,23] that presented data on associations between hearing difficulties and sick leave observed increased risks (OR 4.6; 95\% confidence interval 1.3-16.5 and 1.52 (no confidence interval reported), respectively).

\section{Disability pension}

In all, 66610 individuals were included in the studies with information about disability pension, 63382 with information on sex, of these $64 \%$ were men. The measure of disability pension varied somewhat, both all-cause disability pension $[17,18,32]$ and diagnosis-specific disability pension [29-31] were evaluated. Disability pension was also called ill health retirement [29,31]. The following measures of disability pension were used: granted disability pension $[17,18]$, granted ill health retirement due to hearing loss [29], proportions of pensions compensating for hearing loss [30], ill health retirement [31], and number of granted disability pensions among different occupational diseases [32]. 
Table 1 Summaries of each of the 17 included studies, regarding included data on sickness absence, presented by type of study (cohort, intervention, cross sectional), in chronological order, by first author

\begin{tabular}{|c|c|c|c|c|c|c|c|}
\hline $\begin{array}{l}\text { First } \\
\text { author } \\
\text { Year } \\
\text { Country }\end{array}$ & Aim of the study & $\begin{array}{l}\text { Study population. } \\
\text { Study participants (\% of } \\
\left.\text { men }\left({ }^{\lambda}\right)\right) \text {. } \\
\text { Mean age (age range). }\end{array}$ & $\begin{array}{l}\text { Hearing } \\
\text { diagnoses/ } \\
\text { symptom }\end{array}$ & $\begin{array}{l}\text { Type of data } \\
\text { Years of } \\
\text { follow-up. } \\
\text { Years of data } \\
\text { collection. }\end{array}$ & $\begin{array}{l}\text { Measures used of: } \\
\text { - Sick leave } \\
\text { - Disability pension }\end{array}$ & $\begin{array}{l}\text { Results, regarding } \\
\text { sickness absence }\end{array}$ & $\begin{array}{l}\text { Results regarding } \\
\text { disability pension }\end{array}$ \\
\hline
\end{tabular}

\section{Prospective cohort studies}

\begin{tabular}{ll}
\hline Carlsson & Investigate relation between sudden \\
{$[16]$} & senorineural hearing loss and quality of \\
2011 & life, psychosocial consequences and
\end{tabular}

2011 life psychosocial consequences and

588 (response rate 63\%).

Sudden

Sweden

audiological rehabilitation.

369 patients $(56 \%$ गे). 277

sensorineural Quata

Questionnaire

-Proportion of

$10 \%$ upheld sick-leave

patients on sick benefits before the

with information about sick

leave.

Mean age $59(13-91)$

\begin{tabular}{ll}
\hline Gustafsson & Assess risk of being granted a disability \\
{$[17]$} & pension among people with sick leave \\
2011 & due to otoaudiological diagnoses
\end{tabular}

$\begin{array}{lll}2011 & \text { due to otoaudiological diagnoses } & 40786(45 \% \text { o }) \text {. } \\ \text { Sweden } & \text { compared to other sickness absentees. } & \text { Age range 16-64. }\end{array}$

231499.

hearing loss 7 years.

leave and grade of

sudden sensorineural

? sick leave.

hearing loss, 29\%

directly after and $27 \%$

after $\geq 7$ years, most of them for full time

Otoaudiological
diagnose as the
reason for sick
leave.

Data from

- No. of individuals

162 men with a new

- No. of individuals with a sick-leave

162 men with a new
sick-leave spells due to

certificates and spells ( $>7$ days) due otoaudiological

fom regaudiological

diagnoses and 1815

diagnoses in 1985.

men with sick-leave

spells due to other

1985-96.

diagnoses.

- No. and rate of 353 women with a new

people granted

disability pension

sick-leave spell due to

otoaudiological

with different

otoaudiological

diagnoses.

with sick-leave spells

due to other diagnoses.

\begin{tabular}{|c|c|c|c|c|c|c|c|}
\hline $\begin{array}{l}\text { Skøien [18] } \\
2008 \\
\text { Norway }\end{array}$ & $\begin{array}{l}\text { Assess incidence of vertigo in long-term } \\
\text { sickness absence and to identify } \\
\text { sociodemographic diagnostic predictors } \\
\text { for transition into disability pension }\end{array}$ & $\begin{array}{l}1939355 \text {. } \\
1020 \text { long-term sickness } \\
\text { absentees, due to vertigo or } \\
\text { dizziness (32\% } 3 \text { ). } \\
\text { Age range } 16-62 \text {. }\end{array}$ & $\begin{array}{l}\text { Vertigo (ICD10: } \\
\text { H82) or } \\
\text { dizziness (ICD10: } \\
\text { N17) diagnoses. }\end{array}$ & $\begin{array}{l}\text { Register data. } \\
5 \text { years } \\
1997-2002 .\end{array}$ & $\begin{array}{l}\text {-No. of people on } \\
\text { long-term sick leave } \\
\text { ( } \geq 8 \text { weeks) due to } \\
\text { vertigo or dizziness } \\
\text { diagnoses } \\
\text { - No. granted } \\
\text { disability pension }\end{array}$ & $\begin{array}{l}282 \text { women were on } \\
\text { long-term sick leave due } \\
\text { to vertigo, among } 920 \\
139 \text { women eligible for } \\
\text { sickness absence } \\
\text { benefits and } 134 \text { men } \\
\text { among } 11019216 \\
\text { eligible. }\end{array}$ & $\begin{array}{l}24 \% \text { of men and } 23 \% \text { of } \\
\text { women obtained a } \\
\text { disability pension, Those } \\
\text { with diagnose ICD10: } \\
\text { H82 had a RR 1.5 (95\% } \\
\text { Cl 1.1-1.9) for disability } \\
\text { pension compared to } \\
\text { those with diagnose } \\
\text { ICD10:N17. }\end{array}$ \\
\hline $\begin{array}{l}\text { Bjerlemo } \\
{[19]} \\
2006 \\
\text { Sweden }\end{array}$ & $\begin{array}{l}\text { Follow the recovery process and } \\
\text { explore the disease impact on sick-leave } \\
\text { in patients with acute unilateral } \\
\text { vestibular loss }\end{array}$ & $\begin{array}{l}\text { All } 44 \text { patients from three } \\
\text { hospitals, } 27 \text { responded } \\
\left(48 \% \jmath^{d}\right) \text {. } \\
19 \text { patients with } \\
\text { information about sick } \\
\text { leave. } \\
\text { Mean age } 52(16-70) \text {. }\end{array}$ & $\begin{array}{l}\text { Unilateral } \\
\text { vestibular loss }\end{array}$ & $\begin{array}{l}\text { Questionnaire } \\
\text { data. } \\
6 \text { months. } \\
?\end{array}$ & -Being on sick leave & $\begin{array}{l}\text { At onset, } 95 \% \text { were on } \\
\text { sick leave, after one } \\
\text { month } 26 \% \text {; and after } \\
6 \text { months } 22 \% \text {. }\end{array}$ & \\
\hline $\begin{array}{l}\text { Andersson } \\
{[27]} \\
2000\end{array}$ & $\begin{array}{l}\text { Examine occupational status after } \\
5 \text { years, related to working hours, sick }\end{array}$ & $\begin{array}{l}189 \text { tinnitus patients }(77 \% \\
\text { response rate }=146)(47 \% \\
\text { đ). }\end{array}$ & Tinnitus & $\begin{array}{l}\text { Medical files } \\
\text { and }\end{array}$ & $\begin{array}{l}\text {-Being sickness } \\
\text { absent at admission }\end{array}$ & $\begin{array}{l}\text { No. of sickness } \\
\text { absentees had }\end{array}$ & \\
\hline
\end{tabular}

RR for disability pension 1.42 (95\% Cl 1.23-1.64) among people on sickleave with diagnoses compared to all other on sick leave, sex. 


\begin{tabular}{|c|c|c|c|c|c|c|c|}
\hline Sweden & $\begin{array}{l}\text { leave, pension, unemployment among } \\
\text { patient with tinnitus. }\end{array}$ & $\begin{array}{l}142 . \\
\text { Mean age } 56.4 \text {, age range } \\
22-83 .\end{array}$ & & $\begin{array}{l}\text { questionnaire } \\
\text { data. } \\
\text { Average } \\
4.9 \text { years (3- } \\
10 \text { years). } \\
1988-1995 .\end{array}$ & $\begin{array}{l}\text { and at follow up } \\
\text { (self reported). }\end{array}$ & $\begin{array}{l}\text { decreased ( } 13 \text { vs. } 6 \\
\text { subjects) at follow up. }\end{array}$ & \\
\hline $\begin{array}{l}\text { Holgers } \\
{[26]} \\
2000 \\
\text { Sweden }\end{array}$ & $\begin{array}{l}\text { Investigate risk factors for severe } \\
\text { tinnitus measured as absence from } \\
\text { work related to tinnitus of more than } \\
\text { one month during the } 18 \text { month } \\
\text { period }\end{array}$ & $\begin{array}{l}172 \text { tinnitus patients, } 127 \\
(74 \%) \text { completed } \\
\text { questionnaire. } \\
79 \text { patients not on old age } \\
\text { pensioned (?). } \\
\text { Mean age }+75, \widehat{0} 52 \text {. }\end{array}$ & Tinnitus & $\begin{array}{l}\text { Questionnaire } \\
\text { and register } \\
\text { data. } \\
18 \text { months. } \\
?\end{array}$ & $\begin{array}{l}\text {-Absent from work } \\
>1 \text { month due to } \\
\text { tinnitus. }\end{array}$ & $\begin{array}{l}18 \text { patients had been } \\
\text { absent from work } \\
>1 \text { month during the } \\
\text { study period. }\end{array}$ & \\
\hline $\begin{array}{l}\text { Starzynski } \\
{[32]} \\
1993 \\
\text { Poland }\end{array}$ & $\begin{array}{l}\text { Occupational diseases and } \\
\text { consequences such as disability pension }\end{array}$ & $\begin{array}{l}86871 \text { cases of } \\
\text { occupational diseases, } 10 \\
278 \text { also with } \\
\text { consequences. } \\
3228 \text { noise induced hearing } \\
\text { loss (?) } \\
?\end{array}$ & $\begin{array}{l}\text { Noise-induced } \\
\text { hearing loss }\end{array}$ & $\begin{array}{l}\text { Register data. } \\
\text { ? } \\
\text { 1990-1992. }\end{array}$ & $\begin{array}{l}\text {-No. granted } \\
\text { disability pension } \\
\text { among individuals } \\
\text { with different } \\
\text { occupational } \\
\text { diseases }\end{array}$ & & $\begin{array}{l}\text { Among individuals with } \\
\text { the occupational } \\
\text { disease: noise induced } \\
\text { hearing loss, } 1990(62 \%) \\
\text { had been granted } \\
\text { disability pension. }\end{array}$ \\
\hline \multicolumn{8}{|c|}{ Retrospective cohort studies } \\
\hline $\begin{array}{l}\text { Hagberg } \\
{[21]} \\
2005 \\
\text { Sweden }\end{array}$ & $\begin{array}{l}\text { To determine the incidence of tinnitus, } \\
\text { hearing impairment and } \\
\text { musculoskeletal disorders among } \\
\text { musicians, and the relation to practicing } \\
\text { hours and instrument type. }\end{array}$ & $\begin{array}{l}655 \text { musicians (dropout due } \\
\text { to: death } 2 \% \text {, emigration } \\
6 \% \text {, or non response } 29 \%) \text {. } \\
407 \text { participants }\left(54 \% \jmath^{7}\right) \\
\\
\text { Men: mean age } 35(23-49) \\
\text { Women: mean age } 34 \text { (24- } \\
\text { 57). }\end{array}$ & $\begin{array}{l}\text { Tinnitus, hearing } \\
\text { impairment }\end{array}$ & $\begin{array}{l}\text { Questionnaire } \\
\text { data. } \\
\text { Questions } \\
\text { regarding } \\
\text { present, } \\
\text { previous year, 5, } \\
10 \text {, and } 15 \text { years } \\
\text { ago. } \\
2000\end{array}$ & $\begin{array}{l}\text {-No. having been on } \\
\text { sick-leave due to } \\
\text { hearing problems } \\
\text { (tinnitus and/or } \\
\text { impaired hearing) }\end{array}$ & $\begin{array}{l}13 \text { individuals had been } \\
\text { on sick leave due to } \\
\text { hearing problems. }\end{array}$ & \\
\hline $\begin{array}{l}\text { Sewell [30] } \\
2004 \\
\text { USA }\end{array}$ & $\begin{array}{l}\text { Examine the prevalence of hearing loss } \\
\text { among Union Army veterans in the US } \\
\text { by year, birth cohort and occupation } \\
\text { and to compare civil war pension and } \\
\text { contemporary disability programs by } \\
\text { examining monthly dollar award. }\end{array}$ & $\begin{array}{l}\text { Random sample of US } \\
\text { Army veterans } n=35747 \\
(100 \% \hat{\jmath}) \text {. } \\
17722 \text { receiving a pension. } \\
?\end{array}$ & $\begin{array}{l}\text { Hearing loss } \\
\text { from physical } \\
\text { examination } \\
\text { records }\end{array}$ & $\begin{array}{l}\text { Medical records. } \\
? \\
\text { 1862-1907. }\end{array}$ & $\begin{array}{l}\text {-Proportions of all } \\
\text { pensions } \\
\text { compensating for } \\
\text { hearing loss. }\end{array}$ & & $\begin{array}{l}\text { Total } 5891 \text { or } 33 \% \text { of the } \\
\text { individuals receiving a } \\
\text { pension received } \\
\text { compensation for } \\
\text { hearing loss. Prevalence } \\
\text { increased with time, age } \\
\text { and later birth cohort. }\end{array}$ \\
\hline $\begin{array}{l}\text { Rudin [28] } \\
1987 \\
\text { Sweden }\end{array}$ & $\begin{array}{l}\text { Long-term effects of otitis media on the } \\
\text { general health, measured hearing loss } \\
\text { and the wellbeing of the subjects. }\end{array}$ & $\begin{array}{l}\text { A population-based sample } \\
\text { of } 945 \text { men born in } 1913 \\
\text { ( } 81 \% \text { response rate), } 292 \\
\text { men born } 1923 \text { ( } 75 \% \\
\text { response rate). } \\
927\left(100 \%{ }^{2}\right) \text {. } \\
\text { Age } 60 \text { and } 50 \text { years-old. }\end{array}$ & $\begin{array}{l}\text { History of otitis } \\
\text { media (self- } \\
\text { reported). }\end{array}$ & $\begin{array}{l}\text { Data from } \\
\text { interviews and } \\
\text { registers. } \\
\text { Register data for } \\
\text { 1955-1973 } \\
1973 .\end{array}$ & $\begin{array}{l}\text {-No. of days and } \\
\text { periods of sickness } \\
\text { benefit. }\end{array}$ & $\begin{array}{l}\text { Non-significant }(p=0.20) \\
\text { difference in number of } \\
\text { days or periods of } \\
\text { sickness benefits } \\
\text { between history of otitis } \\
\text { media compared to no } \\
\text { history. }\end{array}$ & \\
\hline
\end{tabular}




\begin{tabular}{|c|c|c|c|c|c|c|c|}
\hline \multicolumn{8}{|c|}{ Intervention studies } \\
\hline $\begin{array}{l}\text { Gates [22] } \\
2004 . \\
\text { USA. }\end{array}$ & $\begin{array}{l}\text { Evaluate a portable low-intensity } \\
\text { alternating pressure generator in } \\
\text { controlling symptoms of Ménière's } \\
\text { disease. } \\
\text { A randomized, double-blinded, placebo } \\
\text { controlled clinical trial. }\end{array}$ & $\begin{array}{l}62 \text { Ménière' patients, } 30 \text { in } \\
\text { treatment group }\left(33 \% \mathcal{J}^{3}\right) \\
\text { and } 32 \text { in control group } \\
\left(31 \% \hat{\jmath}^{3}\right) \text {. } \\
52(84 \%) \text { patients } \\
\text { participated all } 4 \text { months. } \\
\text { Age range } 33-71 .\end{array}$ & $\begin{array}{l}\text { Ménière's } \\
\text { disease }\end{array}$ & $\begin{array}{l}\text { "Diary"-data. } \\
4 \text { months. } \\
2002-2003 .\end{array}$ & $\begin{array}{l}\text {-No. of sick days } \\
\text { (self-reported) } \\
\text { during follow-up. }\end{array}$ & $\begin{array}{l}\text { Median proportion of } \\
\text { sick days all } 4 \text { months: } \\
\text { treatment }=0.00 \text {, } \\
\text { control }=0.02(p=0.02) \text {. } \\
\text { Baseline mean } \\
\text { proportion of sick days } \\
\text { in the treatment }=0.05 \text {, } \\
\text { and in the control }=0.07 \text {. } \\
\text { At } 4 \text { months, } 0.01 \text { in } \\
\text { both groups. }\end{array}$ & \\
\hline $\begin{array}{l}\text { Bjorne [24] } \\
2003 \\
\text { Sweden }\end{array}$ & $\begin{array}{l}\text { A cost-benefit analysis of reduction in } \\
\text { treatment costs, sick leave and disability } \\
\text { pension among working individuals } \\
\text { with Ménière's disease receiving } \\
\text { treatment of tempemanibular and } \\
\text { cervical spine disorders. }\end{array}$ & $\begin{array}{l}\text { Ménière's patients: } 24 \\
\text { treated, } 24 \text { matched } \\
\text { population-based controls. } \\
4 \text { (17\%) dropped out. } \\
19 \text { patients and } 19 \text { controls } \\
\text { (not on disability pension } \\
\text { or old age pension) ( } 47 \% \\
\text { J). } \\
\text { Mean age 52, age range } \\
29-74 \text {. }\end{array}$ & $\begin{array}{l}\text { Ménière's } \\
\text { disease }\end{array}$ & $\begin{array}{l}\text { Register data. } \\
\\
3 \text { years } \\
1990-92 .\end{array}$ & $\begin{array}{l}\text {-No. of sick-leave } \\
\text { days due to } \\
\text { Ménière's or related } \\
\text { symptoms } 3 \text { years } \\
\text { before and } 3 \text { years } \\
\text { after treatment. }\end{array}$ & $\begin{array}{l}\text { Sick leave reduced from } \\
1536 \text { to } 270 \text { days, before } \\
\text { and after treatment, } \\
\text { compared to from } 8 \text { to } \\
6 \text { days for the control } \\
\text { group. }\end{array}$ & \\
\hline $\begin{array}{l}\text { Joore [25] } \\
2003 \\
\text { Netherlands }\end{array}$ & $\begin{array}{l}\text { To assess benefits in terms of gain in } \\
\text { health-related quality of life and } \\
\text { possible savings in terms of increased } \\
\text { productivity and decreased use of } \\
\text { medical services after hearing aid fitting } \\
\text { in a population of moderately hearing } \\
\text { impaired first-time hearing aid } \\
\text { applicants. }\end{array}$ & $\begin{array}{l}80 \text { hearing impaired } \\
\text { patients. } \\
10 \text { with paid employment } \\
\left(100 \% \mathrm{o}^{2}\right) . \\
?\end{array}$ & Hearing loss. & $\begin{array}{l}\text { Interview data. } \\
25 \text { weeks } \\
?\end{array}$ & $\begin{array}{l}\text {-Absence from work } \\
\text { due to health or due } \\
\text { to hearing } \\
\text { impairment }\end{array}$ & $\begin{array}{l}\text { No reported absence } \\
\text { from work due to } \\
\text { hearing impairment or } \\
\text { other health problems } \\
\text { at baseline or at follow- } \\
\text { up among those } \\
\text { employed. }\end{array}$ & \\
\hline \multicolumn{8}{|c|}{ Cross-sectional studies } \\
\hline $\begin{array}{l}\text { Neuhauser } \\
{[10]} \\
2008\end{array}$ & $\begin{array}{l}\text { Assess burden of dizziness and quantify } \\
\text { contribution of vestibular vertigo and } \\
\text { non-vestibular dizziness }\end{array}$ & $\begin{array}{l}8318 \text { random digit sample } \\
\text { (response rate 52\%) } \\
\text { screened for history of } \\
\text { dizziness; } 1157 \text { fulfilled } \\
\text { criteria (had dizziness). }\end{array}$ & $\begin{array}{l}\text { Vestibular } \\
\text { vertigo. }\end{array}$ & $\begin{array}{l}\text { Interview data. } \\
\text { NA }\end{array}$ & $\begin{array}{l}\text {-Reported sick leave } \\
\text { among working } \\
\text { individuals }\end{array}$ & $\begin{array}{l}\text { Among individuals with } \\
\text { vestibular vertigo, } 40.6 \% \\
\text { reported sick leave } \\
\text { compared to } 14.7 \% \\
\text { among those with non- } \\
\text { vestibular dizziness } \\
p<0.001 \text {. } \\
\text { Hiaher rates among }\end{array}$ & \\
\hline & & $\begin{array}{l}1003(87 \%)(?) \\
\text { Age range } 18-79 .\end{array}$ & & 2003. & & women. & \\
\hline $\begin{array}{l}\text { Ide [29] } \\
2007 \\
\text { UK }\end{array}$ & $\begin{array}{l}\text { Define level of hearing loss associated } \\
\text { with ill-health retirement. }\end{array}$ & $\begin{array}{l}35737 \text { fire fighters. } \\
3366 \text { whole time firefighters } \\
\text { granted ill-health retirement } \\
\left(100 \% \delta^{\lambda}\right) \text {. } \\
\text { Mean age among } \\
\text { firefighters granted ill } \\
\text { health retirement due to } \\
\text { hearing loss: } 49 \text { years, range } \\
29-59 .\end{array}$ & $\begin{array}{l}\text { Hearing loss in } \\
\text { decibels }\end{array}$ & $\begin{array}{l}\text { Questionnaire } \\
\text { and medical } \\
\text { data provided } \\
\text { by work sites. } \\
\text { NA } \\
1997-2002 \\
\text { (60 months). }\end{array}$ & $\begin{array}{l}\text {-No. and rate } \\
\text { granted ill-health } \\
\text { retirement due to } \\
\text { hearing loss. }\end{array}$ & & $\begin{array}{l}4 \%(n=135 \text { among } \\
3366) \text { of the ill health } \\
\text { retirements were due to } \\
\text { hearing loss. }\end{array}$ \\
\hline
\end{tabular}




\begin{tabular}{|c|c|c|c|c|c|c|c|}
\hline $\begin{array}{l}\text { Kramer [20] } \\
2006 \\
\text { Netherlands }\end{array}$ & $\begin{array}{l}\text { Compare the occupational performance } \\
\text { of employees with hearing impairment } \\
\text { and normal hearing. }\end{array}$ & $\begin{array}{l}\text { Unknown } \\
150 \text { hearing impaired } \\
\text { employed }\left(47 \% \partial^{7}\right), 60 \\
\text { normal hearing from the } \\
\text { same workplaces }\left(48 \%{ }^{2}\right) \text {. } \\
\text { Mean age normal hearing } \\
42.7 \text {, impaired hearing } 45.3 \\
(21-65) \text {. }\end{array}$ & $\begin{array}{l}\text { Post lingual } \\
\text { hearing loss }\end{array}$ & $\begin{array}{l}\text { Questionnaire } \\
\text { data. } \\
\text { NA } \\
\text { 2000-2001. }\end{array}$ & $\begin{array}{l}\text {-No. of sick-leave } \\
\text { days in the last } \\
12 \text { months and } \\
\text { reason for sick leave. }\end{array}$ & $\begin{array}{l}\text { Hearing impaired had } \\
\text { increased risk of sick } \\
\text { leave due to distress/ } \\
\text { strain OR } 4.6 \text { (1.3-16.5) } \\
\text { compared to normal } \\
\text { hearing, adjusted for } \\
\text { age, sex, education, job } \\
\text { demands/control/ } \\
\text { support, career } \\
\text { satisfaction and type of } \\
\text { contract. }\end{array}$ & \\
\hline $\begin{array}{l}\text { Chau [23] } \\
2004 \\
\text { France }\end{array}$ & $\begin{array}{l}\text { Assess relationships of job, age and life } \\
\text { conditions with causes and severity of } \\
\text { occupational injuries. }\end{array}$ & $\begin{array}{l}880 \text { construction workers } \\
\text { (employed since at least } \\
5 \text { years) with non-fatal } \\
\text { accident and sick-leave } \\
\text { occasion and been seen by } \\
\text { the physician. } \\
880\left(100 \%{ }^{2}\right) \text {. } \\
\text { ? }\end{array}$ & $\begin{array}{l}\text { Hearing } \\
\text { impairment }\end{array}$ & $\begin{array}{l}\text { Questionnaire } \\
\text { data (filled in by } \\
\text { the company } \\
\text { physician). } \\
\text { NA } \\
\text { 1995-96. }\end{array}$ & $\begin{array}{l}\text {-Sick leave }>60 \text { days, } \\
\text { reported by } \\
\text { occupational } \\
\text { physician. }\end{array}$ & $\begin{array}{l}\text { Hearing impaired } \\
\text { individuals had a higher } \\
\text { risk of sick leave } \\
\text { (OR=1.52). Analysis } \\
\text { adjusted for age, BMl, } \\
\text { sleep disorders, smoking, } \\
\text { sporting activities, and } \\
\text { occupation. }\end{array}$ & \\
\hline $\begin{array}{l}\text { Ide [31] } \\
1998 \text { UK }\end{array}$ & $\begin{array}{l}\text { Count the causes of death and ill-health } \\
\text { retirement, determine the emergence } \\
\text { of any trends and examine the } \\
\text { relationships between length of service } \\
\text { and cause of ill health retirement or } \\
\text { death among fire-fighters }\end{array}$ & $\begin{array}{l}488(=96.6 \% \text { with clinical } \\
\text { notes) }(100 \% \text { ふ). } \\
\text { Mean age } 43.75\end{array}$ & $\begin{array}{l}\text { Ear and mastoid } \\
\text { diagnoses } \\
\text { (ICD9:380-389) } \\
\text { as primary } \\
\text { diagnose. }\end{array}$ & $\begin{array}{l}\text { Data from } \\
\text { annual reports, } \\
\text { management } \\
\text { information } \\
\text { system and } \\
\text { medical records. } \\
\text { NA } \\
\text { 1985-1994 }\end{array}$ & $\begin{array}{l}\text {-No. with ill-health } \\
\text { retirement }\end{array}$ & & $\begin{array}{l}16(3 \%) \text { of the ill-health } \\
\text { retirements were due to } \\
\text { ear and mastoid } \\
\text { diagnoses. }\end{array}$ \\
\hline
\end{tabular}




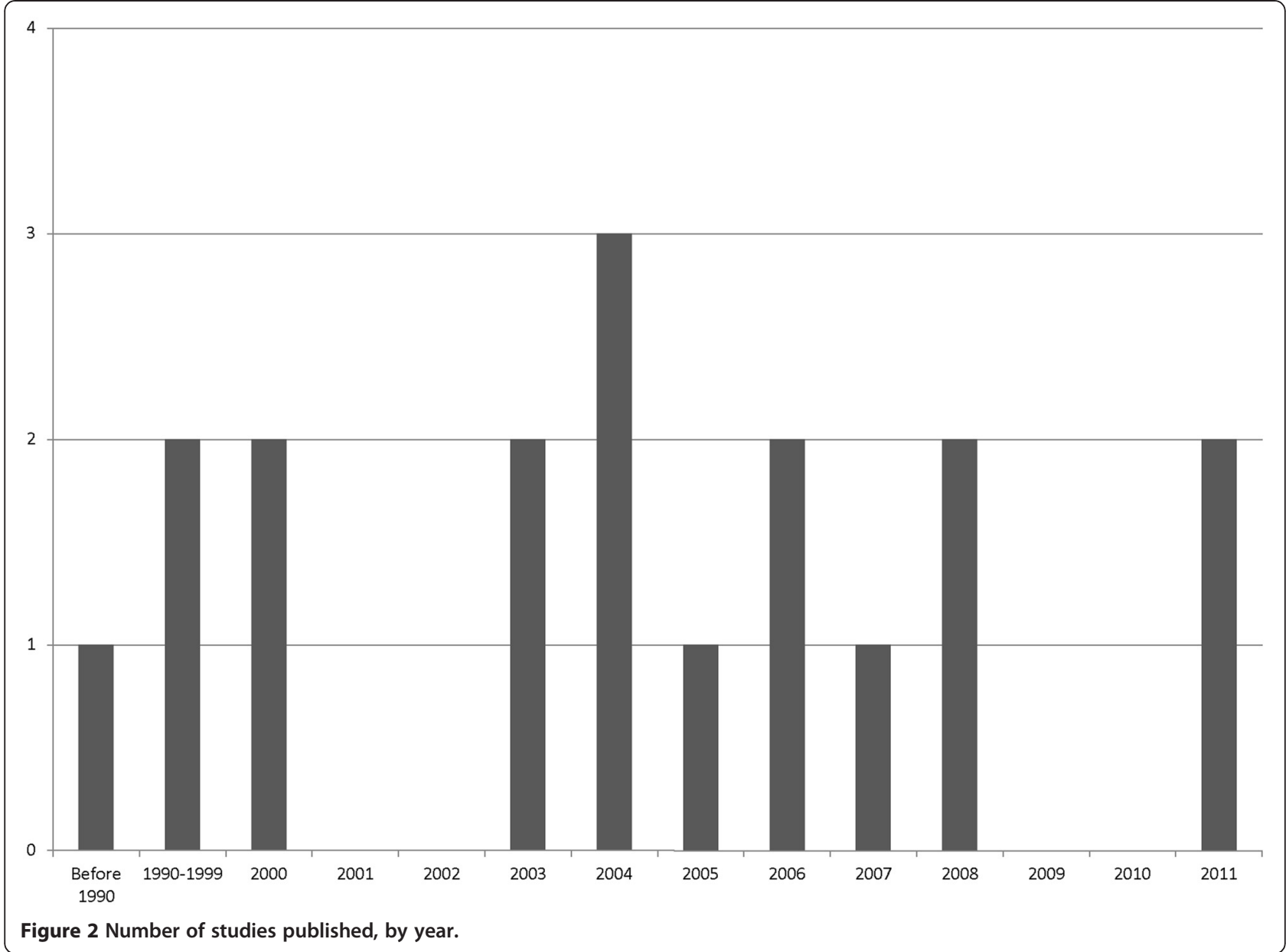

The two studies presenting data on associations between hearing diagnoses as the reason for sick leave [17] or vertigo [18] and disability pension both observed an increased risk (RR 1.42; 95\% CI 1.23-1.64 and RR 1.5; 95\% CI 1.1-1.9, respectively).

\section{Discussion and conclusions}

The aim of this literature review was to summarize research results from published studies that present empirical data on hearing difficulties or other ear-related diagnoses and sick leave or disability pension. The most striking finding from this literature review is the low number of published studies about sickness absence or disability pension due to hearing difficulties/diagnoses, considering how prevalent such hearing difficulties are. Secondly, the vast incongruity of the results, which partly can be explained by the large amount of different exposures, outcomes and measures examined in the different studies. We could only identify 18 relevant studies to include in this review, in spite of using several different search methods. Moreover, there are remarkable few relevant studies published on associations (i.e. rate ratios or odds ratios), between hearing difficulties and sick leave $[20,23]$ or disability pension $[17,18]$, however, all the four studies with such data, reported positive associations. Only two studies reported data stratified by gender, both showing a higher risk of disability pension among women with sick-leave due to audiological [17] or vertigo [18] diagnoses compared to men. We found no studies that assessed hearing difficulties as an outcome, after, for example, sick-leave or disability pension. The extremely large variation of data on hearing difficulties and on sickness absence and disability pension means that, at this stage, it is not constructive to compare results from the different studies regarding risks for sick leave or disability pension.

The strengths of this study are the systematic approach in searching and assessing studies, including a large number of keywords and the employing of a wide search strategy in well-known literature databases. Another strength is that two researchers independently conducted the searches. The very wide search made it possible to identify studies about hearing difficulties or other ear-related diagnoses, sick leave, and disability pension from different scientific disciplines. However, we cannot rule out that we might have missed studies; 
Table 2 Categorization of included studies

\begin{tabular}{|c|c|c|c|c|c|c|c|c|}
\hline \multirow[t]{2}{*}{ First author, year } & \multicolumn{3}{|c|}{ Study design } & \multicolumn{3}{|c|}{ Study group } & \multicolumn{2}{|c|}{ Outcome } \\
\hline & Cohort & Intervention & $\overline{\text { Cross-sectional }}$ & Population & Employed & Patients & Sick leave & Disability pension \\
\hline Carlsson [16], 2011 & $x$ & & & & & $x$ & $x$ & \\
\hline Gustafsson [17], 2011 & $x$ & & & $x$ & & & $x$ & $x$ \\
\hline Neuhauser [10], 2008 & & & $x$ & $x$ & & & $x$ & \\
\hline Skøien [18], 2008 & $x$ & & & $x$ & & & $x$ & $x$ \\
\hline Ide [29], 2007 & & & $x$ & & $x$ & & & $x$ \\
\hline Bjerlemo [19], 2006 & $x$ & & & & & $x$ & $x$ & \\
\hline Kramer [20], 2006 & & & $x$ & & $x$ & & $x$ & \\
\hline Hagberg [21], 2005 & $x$ & & & & $x^{*}$ & & $x$ & \\
\hline Gates [22], 2004 & & $x$ & & & & $x$ & $x$ & \\
\hline Chau [23], 2004 & & & $x$ & & $x$ & & $x$ & \\
\hline Sewell [30], 2004 & $x$ & & & & $x$ & & & $x$ \\
\hline Bjorne [24], 2003 & & $x$ & & & $x$ & $x$ & $x$ & \\
\hline Joore [25], 2003 & & $x$ & & & & $x$ & $x$ & \\
\hline Andersson [27], 2000 & $x$ & & & & & $x$ & $x$ & \\
\hline Holgers [26], 2000 & $x$ & & & & & $x$ & $x$ & \\
\hline Ide [31], 1998 & & & $x$ & & $x$ & & & $x$ \\
\hline Starzynski [32], 1993 & $x$ & & & & $x$ & & & $x$ \\
\hline Rudin [28], 1987 & $x$ & & & $x$ & & & $x$ & \\
\hline
\end{tabular}

some scientific journals are not included in the literature databases; for example, newly established scientific journals. Moreover, several studies only reported the information without this being the actual aim of the study in question, which increase the risk that we might have missed some studies. Furthermore, the risk of publication

Table 3 Hearing difficulties in relation to sick leave and disability pension, categorization of included studies according to data reported

\begin{tabular}{|c|c|c|}
\hline & Data on sick leave & $\begin{array}{l}\text { Data on disability } \\
\text { pension }\end{array}$ \\
\hline \multirow{6}{*}{$\begin{array}{l}\text { Sick leave or disability } \\
\text { pension due to } \\
\text { hearing difficulties }\end{array}$} & Gustafsson [17], 2011 & Ide [29], 2007 \\
\hline & Skøien [18], 2008 & Sewell [30], 2004 \\
\hline & Hagberg [21], 2005 & Ide [31], 1998 \\
\hline & Bjorne [24], 2003 & \\
\hline & Joore [25], 2003 & \\
\hline & Holgers [26], 2000 & \\
\hline \multirow{8}{*}{$\begin{array}{l}\text { Sick leave or disability } \\
\text { pension, irrespective } \\
\text { of diagnoses }\end{array}$} & Carlsson [16], 2011 & Gustafsson [17], 2011 \\
\hline & Neuhauser [10], 2008 & Skøien [18], 2008 \\
\hline & Bjerlemo [19], 2006 & Starzynski [32], 1993 \\
\hline & Gates [22], 2004 & \\
\hline & Chau [23], 2004 & \\
\hline & Joore [25], 2003 & \\
\hline & $\overline{\text { Andersson [27], } 2000}$ & \\
\hline & Rudin [28], 1987 & \\
\hline
\end{tabular}

Note: the study by Kramer [20], 2006 reported sick leave due to distress/strain, i.e. not sick leave in general nor due to hearing difficulties. bias cannot be ignored, small studies showing no associations or inverse associations might not have been published at all. Nevertheless, the number of retrieved publications with this wide search strategy resulted in very few relevant studies which indicate that sick leave and disability pension with hearing difficulties or other ear-related diagnoses have not been widely studied in scientific contexts.

In areas where more studies have been conducted, usually only studies that have a minimum level of scientific quality are included in systematic reviews. It became evident that the number of conducted studies in this field did not allow for excluding studies of lower scientific quality. Instead, the aim was to use all available studies to gain knowledge, why all studies identified were included, irrespective of quality.

In conclusion, there are remarkably few studies published on associations between hearing difficulties or other ear-related diagnoses and sick leave and/or disability pension and there are very large variations between the studies regarding design, study groups, analyses, and outcome measures. Nevertheless, all studies that disclosed results on associations between hearing difficulties or other ear-related diagnoses and sick leave or disability pension reported positive associations. However, at this stage, these results cannot be considered to provide enough evidence for such associations. This finding warrants further attention and not only are more, but also better studies needed before any 
conclusions can be made. There is an overall lack of prospective studies with long follow-up investigating the risk of diagnosis specific sick leave and disability pension among individuals with hearing difficulties or other earrelated diagnoses. Also intervention studies targeting the workplaces more directly would be of great interest.

\section{Competing interest}

The authors declare that they have no conflict of interest.

\section{Authors' contributions}

Study concept and design: EF, KA. Systematic literature search: EF, KG. Interpretations of data: EF, KG, KA. Drafting of the manuscript: EF. Critical revision of the manuscript for important intellectual content: KG, KA. All authors have read and approved final version of manuscript.

\section{Acknowledgement}

This study was financed by the Swedish Council for Working Life and Social Research (FAS by Swedish acronym) Center program Hearing Disabilities in Working Life and Society. The funders had no role in study design, data collection and analysis, decision to publish, or preparation of the manuscript.

Received: 4 April 2012 Accepted: 6 September 2012

Published: 12 September 2012

\section{References}

1. Kochkin S, Trak M VIl: Hearing loss population tops 31 million people. Hearing-impaired population continues to increase - along with satisfaction ratings for hearing instruments. Hearing Review 2005, 12:16-29.

2. Wallhagen Ml, Strawbridge W, Cohen RD, Kaplan GA: An increasing prevalence of hearing impairment and associated risk factors over three decades of the Alameda County Study. Am J Public Health 1997, 87:440-442.

3. Agrawal Y, Platz EA, Niparko J: Prevalence of hearing loss and differences by demographic characteristics among US adults: data from the National Health and Nutrition Examination Survey, 1999-2004. Arch Intern Med 2008, 168:1522-1530.

4. Mathers $C D$, Loncar D: Projections of global mortality and burden of disease from 2002 to 2030. PLoS Med 2006, 3:2011-2030

5. Sorri M, Roine R: Age-adjusted prevalence of hearing impairment has significantly increased during the last two decades. Scand Audiol Suppl 2001, 54:5-7.

6. Alexanderson K, Norlund A: Swedish Council on Technology Assessment in Health Care (SBU). Chapter 2. Methods used for the systematic literature search and for the review of relevance, quality, and evidence of studies. Scand J Public Health Suppl 2004, 63:31-35.

7. Rosenhall U, Jonsson R, Soderlind O: Self-assessed hearing problems in Sweden: a demographic study. Audiology 1999, 38:328-334.

8. Hasson D, Theorell T, Westerlund H, Canlon B: Prevalence and characteristics of hearing problems in a working and non-working Swedish population. J Epidemiol Community Health 2010, 64:453-460.

9. Hörselskadades Riksförbund. Arrrapport. (The Swedish Association of Hard of Hearing People, yearly report). (In Swedish). 2008. http://www.hrf.se/ templates/kampanj.aspx?id=2284\&baseRef=2284. Accessed September 2012.

10. Neuhauser HK, Radtke A, von Brevern M, Lezius F. Feldmann M, Lempert T: Burden of dizziness and vertigo in the community. Arch Intern Med 2008, 168:2118-2124

11. Alexanderson K, Norlund A: Sickness absence - causes, consequences, and physicians sickness cerification practice. A systematic literature review by the Swedish Council on Technology Assessment in Health Care. Scand J Public Health 2004, 32(Suppl 63):1-263.

12. Marmot M, Feeney A, Shipley M, North F, Syme SL: Sickness absence as a measure of health status and functioning: from the UK Whitehall II study. J Epidemiol Community Health 1995, 49:124-130.

13. Alexanderson K: Work and III-health among Women and Men in Sweden. In Worklife and Health in Sweden 2000. Edited by Marklund S. Stockholm: National Institute for Working Life; 2001

14. Liberati A, Altman DG, Tetzlaff J, Mulrow C, Gotzsche PC, loannidis JP, Clarke M, Devereaux PJ, Kleijnen J, Moher D: The PRISMA statement for reporting systematic reviews and meta-analyses of studies that evaluate health care interventions: explanation and elaboration. PLoS Med 2009, 6:e1000100

15. International Statistical Classification of Diseases and Related Health Problems, Tenth Revision (ICD-10).World Health Organization (WHO). 1992. http://apps. who.int/classifications/icd10/browse/2010/en. Accessed September 2012.

16. Carlsson PI, Hall M, Lind KI, Danermark B: Quality of life, psychosocial consequences, and audiological rehabilitation after sudden sensorineural hearing loss. Int I Audiol 2011, 50:139-144.

17. Gustafsson K, Backenroth-Ohsako G, Rosenhall U, Ternevall-Kjerulf E, Ulfendahl M, Alexanderson K: Future risk for disability pension among people with sickness absence due to otoaudiological diagnoses: a population-based cohort study with a 12-year follow-up. Scand J Public Health 2011, 39:501-507.

18. Skooien AK, Wilhelmsen $\mathrm{K}$, Gjesdal S: Occupational disability caused by dizziness and vertigo: a register-based prospective study. British Journal of General Practice 2008, 58:619-623.

19. Bjerlemo B, Kollén L, Boderos I, Kreuter M, Möller C: Recovery after early vestibular rehabilitation in patients with acute unilateral vestibular loss. Audiological Medicine 2006, 4:117-123.

20. Kramer SE, Kapteyn TS, Houtgast T: Occupational performance: comparing normally-hearing and hearing-impaired employees using the Amsterdam checklist for hearing and work. Int J Audiol 2006, 45:503-512

21. Hagberg $M$, Thiringer $G$, Brandstrom L: Incidence of tinnitus, impaired hearing and musculoskeletal disorders among students enrolled in academic music education-a retrospective cohort study. Int Arch Occup Environ Health 2005, 78:575-583.

22. Gates GA, Green JD Jr, Tucci DL, Telian SA: The effects of transtympanic micropressure treatment in people with unilateral Meniere's disease. Arch Otolaryngol Head Neck Surg 2004, 130:718-725.

23. Chau N, Gauchard GC, Siegfried C, Benamghar L, Dangelzer JL, Francais M, Jacquin R, Sourdot A, Perrin PP, Mur JM: Relationships of job, age, and life conditions with the causes and severity of occupational injuries in construction workers. Int Arch Occup Environ Health 2004, 77:60-66.

24. Bjorne A, Agerberg G, Bjorne A, Agerberg G: Reduction in sick leave and costs to society of patients with Meniere's disease after treatment of temporomandibular and cervical spine disorders: a controlled six-year cost-benefit study. Cranio 2003, 21:136-143.

25. Joore MA, Brunenberg DEM, Chenault MN, Anteunis LC: Societal effects of hearing aid fitting among the moderately hearing impaired. International Journal of Audiology 2003, 42:152-160.

26. Holgers KM, Erlandsson SI, Barrenas ML: Predictive factors for the severity of tinnitus. Audiology 2000, 39:284-291.

27. Andersson G: Longitudinal follow-up of occupational status in tinnitus patients. Int Tinnitus J 2000, 6:127-129.

28. Rudin R, Svardsudd K: Otitis media and well-being in a male population in Gothenburg. The study of men born in 1913. Acta Otolaryngol 1987, 104:454-462.

29. Ide C: Hearing loss, accidents, near misses and job losses in firefighters. Occup Med (Lond) 2007, 57:203-209.

30. Sewell RK, Song C, Bauman NM, Smith RJ, Blanck P: Hearing loss in Union Army veterans from 1862 to 1920. Laryngoscope 2004, 114:2147-2153.

31. Ide CW: Failing firefighters: a survey of causes of death and ill-health retirement in serving firefighters in Strathclyde, Scotland from 1985-94. Occup Med (Lond) 1998, 48:381-388.

32. Starzynski Z, Izycki J: Occupational diseases in Poland during the years 1984-1992. Pol J Occup Med Environ Health 1993, 6:299-308.

doi:10.1186/1471-2458-12-772

Cite this article as: Friberg et al.: Hearing difficulties, ear-related diagnoses and sickness absence or disability pension - a systematic literature review. BMC Public Health 2012 12:772. 